\title{
TEMA 3-2014: LINFEDEMA Y GÁNGER DE MAMA
}

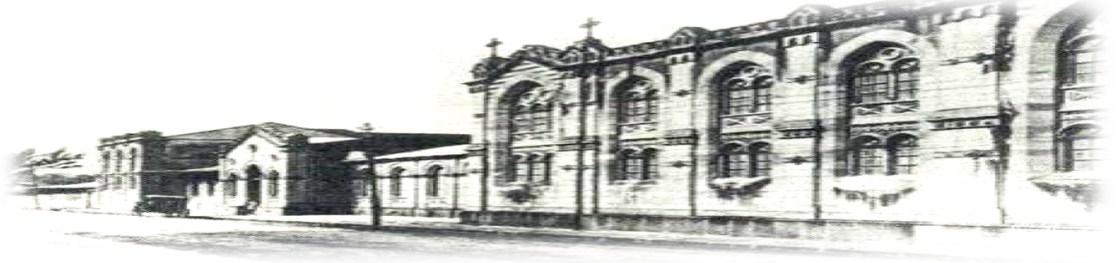

Hospital San quan de Dias. San José, Costa Rica. Fundado en 1845

Recibido: 27/02/2014

Aceptado: $\quad 26 / 03 / 2014$

Andrea Medina Méndez ${ }^{1}$

Vanessa Úcles ${ }^{2}$

${ }^{1}$ Médica Residente de Medicina Paliativa de Adultos de la UCR-CENDEISSS. CCSS Social Costa Rica. Correo electrónico: andreamedinacr@hotmail.com

${ }^{2}$ Médica Fisiatra. Msc Medicina Paliativa del servicio de Fisiatría y Rehabilitación del Hospital Dr. Calderón Guardia. Profesora del postgrado de Medicina Paliativa de Adultos de la UCR-CENDEISSS. San José. Costa Rica.

\section{RESUMEN}

El Cáncer de Mama (CM) constituye el 17\% de los tumores y el 14\% de las muertes por cáncer entre las mujeres costarricenses. En los países desarrollados la causa más frecuente de linfedema es aquel secundario a neoplasias, sus complicaciones y sus tratamientos ${ }^{(1)}$. El linfedema se define como la tumefacción causada por una acumulación de líquido en el intersticio ${ }^{(2)}$ y no existe un criterio único y consensuado para hacer el diagnóstico ${ }^{(3)}$. Su tratamiento es difícil, costoso y consume tiempo ya que es necesario un manejo interdisciplinario ${ }^{(4)}$. Además, no hay un consenso para su tratamiento específico; tampoco existen protocolos terapéuticos claros ${ }^{(3)}$. Conclusiones: el linfedema es una patología frecuente en las pacientes de CM tratadas, que con una adecuada rehabilitación puede prevenirse y en ocasiones revertirse, mejorando así sustancialmente la calidad de vida de las pacientes. Recomendaciones: es fundamental la referencia oportuna a los servicios de rehabilitación de las pacientes mastectomizadas para la adecuada prevención y/o tratamiento del linfedema independientemente de su estadio tumoral.

\section{PALABRAS CLAVE}

Linfedema. Cáncer de mama. Prevención.

\section{ABSTRACT}

Breast Cancer counts for the $17 \%$ of tumors and $14 \%$ of cancer deaths among women in Costa Rica. In developed countries, the most common cause of lymphedema are secondary to neoplasms, complications and treatments ${ }^{(1)}$. Lymphedema is defined as the swelling caused by a buildup of fluid in the interstitium ${ }^{(2)}$. There is no single, agreed criteria for making the diag$\operatorname{nosis}^{(3)}$. Treatment is difficult, expensive and time consuming and nterdisciplinary management is required ${ }^{(4)}$. There is no consensus on specific treatment for lymphedema. There are no clear treatment protocols ${ }^{(3)}$. Conclusions: Lymphedema is a common condition in breast cancer patients and treated with proper rehabilitation that can be prevented and sometimes reversed substantially improving the quality of life of patients. Recommendations: Timely referral to rehabilitation services for patients who under- 
went mastectomy for adequate prevention and/or treatment of lymphedema regardless of tumor stage.

\section{KEY WORDS}

Lymphedema. Breast cancer. Prevention.

\section{INTRODUCCIÓN}

La incidencia de Cáncer de Mama (CM) en Costa Rica (CR) se ha incrementado durante los últimos veinte años, con un aumento del $65 \%$ de nuevos casos en este periodo. Por otro lado, el riesgo de padecer o morir por cáncer de mama en Costa Rica ha aumentado en forma directamente proporcional con la edad ${ }^{(5)}$. El CM constituye el $17 \%$ de los tumores y el $14 \%$ de las muertes por cáncer entre las mujeres costarricenses.

Sin embargo, a pesar de que el CM es el cáncer que más muertes provoca en la población costarricense femenina; hay un porcentaje de pacientes que sobreviven, las cuales vivirán con las secuelas del tratamiento brindado para su curación, por lo que deben recibir un seguimiento adecuado, en aras de prevenir otras complicaciones asociadas como el linfedema de miembros superiores.

\section{DISCUSIÓN}

\section{Generalidades}

El linfedema es una patología crónica y se caracteriza principalmente por cuatro componentes: edema, exceso de proteínas, inflamación crónica y fibrosis ${ }^{(6)}$. En el mundo, la causa más frecuente es la filariasis ${ }^{(1)}$. El linfedema secundario a neoplasias (sus complicaciones o tratamiento propiamente dicho) es el tipo más frecuente en los países desarrollados ${ }^{(1)}$. Se considera que es una condición subdiagnosticada y subtratada, que afecta a muchos sobrevivientes del cáncer ${ }^{(7,8)}$.

La incidencia del linfedema del miembro su perior (MS) va del 6 al $70 \%{ }^{(9)}$ ó del 10 al 34\% ${ }^{(10)}$ dependiendo de la revisión. Esta gran diferencia en rangos se relaciona a los diferencias en el tratamiento del CM, método de medición, atraso en la medición y la definición de linfedema que se utilice.
Lamentablemente, a nivel internacional se documenta, que los pacientes con linfedema de MS son referidos a unidades especializadas de linfedema hasta 2.5 años después de iniciados los síntomas $^{(11)}$. Los objetivos del tratamiento rehabilitador son prevenir la progresión del linfedema, disminuir o mantener el tamaño del MS, aliviar el disconfort, prevenir episodios recurrentes de celulitis y educar al paciente sobre el antocuidado-automanejo ${ }^{(9,10)}$.

\section{Fisiopatología del linfedema}

La linfa es un líquido semejante a la sangre pero sin hematíes. El sistema linfático devuelve a la sangre proteínas y lípidos no utilizados por las células.

En el MS, la linfa de esta región, así como la de la región externa de la mama drena en los linfáticos axilares. Los linfáticos de la mano drenan en la axila tras pasar por los ganglios cubitales a nivel del codo; no obstante, el dedo pulgar, el índice y el dorso de la mano drenan directamente, sin pasar por esta estación linfática intermedia. Una pequeña parte de la linfa procedente del MS, un $10 \%$, no drena en la axila, sino por la región posterior y anterior deltoidea ${ }^{(1)}$.

$$
\text { ¿Qué es el linfedema? }
$$

Se define como la tumefacción causada por una acumulación de líquido en el intersticio. La inflamación crónica y la fibrosis son causadas por la proliferación secundaria de neutrófilos, macrófagos, fibroblastos y la acumulación de colágeno $^{(13)}$. El sistema linfático como barrera frente a las infecciones también se va altera debido a la $\operatorname{estasis}^{(1)}$.

\section{Cuadro Clínico}

En los periodos iniciales, hay sensación de edema y disconfort moderado en el miembro afectado, que puede progresar a una limitación articular, dolor y decoloración de la piel, con aumento del riesgo de infección ${ }^{(14)}$. También se puede experimentar ansiedad, depresión, aislamiento social y problemas sexuales ${ }^{(16)}$. Usualmente es indoloro, pero la paciente sí puede percibir disconfort, tensión, parestesias o sensación de pesadez, que en algunos casos se describe como "dolor" si bien no suele ser severo. Normalmente el dolor asociado al linfedema es más bien una complicación $^{(8)}$. 
El signo de Kaposi-Stemmer (o de Stemmer) es un signo patognomónico que consiste en la imposibilidad de pinzar la piel del dorso del segundo dedo del pie o la mano. Un falso negativo de este signo no excluye un linfedema, pero para algunos autores no existe la posibilidad de un falso positivo ${ }^{(9)}$.

\section{Diagnóstico}

No existe un criterio único y consensuado ${ }^{(16)}$ y el diagnóstico del linfedema generalmente se hace en los primeros 18 meses postquirúrgico del $\mathrm{CM}^{(1)}$.

El linfedema aparecerá clínicamente cuando el volumen acumulado de linfa en el intersticio supere aproximadamente el $20 \%$ del normal ${ }^{(1)}$. Se considera que el cambio en $\geq 200 \mathrm{ml}$ en el volumen; $\geq 2 \mathrm{~cm}$ en la circunferencia en más de dos medidas adyacentes ${ }^{(17)} y \geq 10 \%$ de cambio de volumen en el miembro afectado en la perimetría son diagnósticos.

Clínicamente el edema del linfedema es duro, asimétrico y con signo de Stemmer positivo ${ }^{(2)}$.

\section{Diagnóstico diferencial:}

Causas sistémicas: insuficiencia cardiaca congestiva, hipoalbuminemia, insuficiencia renal, síndrome nefrótico y hepatopatía avanzada ${ }^{(11)}$.

Causas locales: enfermedad venosa crónica, lipedema (adiposalgia o lipomatosis dolorosa), trombosis venosa profunda, linfangiosarcoma o enfermedad de Steward-Treves ${ }^{(8,12)}$.

\section{Estudios diagnósticos}

Se recomienda realizar un análisis de sangre general para descartar causas sistémicas, determinar el valor de proteínas séricas y de hormonas tiroideas $^{(18)}$.

Sólo la linfografía radioisotópica y la linfografía con contraste son estudios específicas del sistema linfático; el resto sirve indirectamente tras valorar el estado arterial, venoso y micro circulatorio $^{(6)}$.

Técnicas de

Medición del miembro superior:

\section{Circometría.}

La circometría es un método sencillo, inocuo y válido para establecer el diagnóstico de linfedema así como para controlar su evolución ${ }^{(8)}$. Consiste en la medición de múltiples circunferencias desde un punto fijo. A través de ella se puede hacer una estimación indirecta de la extremidad basándose en la fórmula del cono truncado.

\section{Volumen}

La medición del volumen de agua desplazada se lleva a cabo sumergiendo la extremidad superior en un cilindro que contiene un volumen conocido de agua. La cantidad de agua desplazada por el brazo es equivalente a su volumen ${ }^{(6)}$.

La volumetría optoelectrónica mide el volumen y la forma de la extremidad mediante tecnología láser de infrarrojos, realizando lecturas cada 4 $\mathrm{cm}$ a lo largo de la extremidad ${ }^{(16)}$. Calcular el volumen del edema a partir de 2 mediciones de la circunferencias es el método de Herpertz ${ }^{(19)}$.

Todos los aparatos optoelectrónicos de cálculo del volumen se han considerado la prueba volumétrica "gold standard,"(20) siendo el único método de medición en el que no existen prácticamente diferencias entre la variabilidad interobservador e intraobservador. Es un método caro, poco extendido y con algunas deficiencias que complican la exploración.

\section{Bioimpedancia}

La bioimpedancia es un método para el diagnóstico precoz o para la valoración de líquido extracelular, que mide la resistencia del cuerpo al paso de una corriente eléctrica de baja frecuencia. Es un método caro, no solo por su coste, sino por la necesidad de un operador para el manejo de datos y el cálculo de la curva. No cuantifica el volumen y se necesitan más estudios que avalen uso y confirmen su fiabilidad y precisión ${ }^{(16)}$.

\section{Otros}

La tonometría intenta medir la resistencia de los tejidos a la compresión, pudiendo identificar acúmulos de líquido o fibrosis pulmonar. En la página web de la Sociedad española de Medicina Física y Rehabilitación se encuentra disponible la "calculadora de linfedema" 


\section{Factores de riesgo}

Los factores de riesgo se pueden clasificar:

\section{Factores relacionados con el tratamien- to}

La mastectomía, la radiación a la axila, DGA y la extirpación de un alto número de ganglios linfá$\operatorname{ticos}^{(21)}$, la extensión de la DGA y la radioterapia posterior a la cirugía, se consideran los mayores factores de riesgo ${ }^{(6,22)}$.

\section{Factores relacionados con la enferme- dad}

Un estadio avanzado, tamaño tumoral y localización tumoral en cuadrante supero externo de la mama son considerados de mayor riesgo ${ }^{(6)}$.

\section{Otros}

La obesidad ${ }^{(13)}$ con un Índice de Masa Corporal $($ IMC) $\geq 30$ tienen alto riesgo de desarrollar linfedema ${ }^{(22,23)}$, al momento del diagnóstico, o si la paciente sube de peso después del diagnóstico. Hay evidencia de que un IMC menor a $25 \mathrm{~kg} / \mathrm{m}^{2}$ y una dieta pobre en grasas previene el linfede$\mathrm{ma}^{(24)}$.

Los factores de riesgo conocidos para linfedema secundario, mencionados anteriormente, solo explican parcialmente quiénes lo desarrollarán posterior al cáncer, lo que sugiere que la susceptibilidad genética puede influir ${ }^{(25)}$.

\section{Clasificación}

Existen varias maneras de clasificar el linfedema:

\section{Según su Evolución:}

Agudo o transitorio: un episodio único de edema que dura menos de 3 meses y desaparece con o sin tratamiento ${ }^{(26)}$.

Crónico o persistente: cuando dura más de 3 meses $^{(26)}$.

\section{Según su Etiología}

Primario: por un defecto en el sistema linfático y a su vez se sub divide en aplasia, hipoplasia e hiperplasia linfática y puede presentarse desde el nacimiento o en la segunda y tercera década de vida. Representa cerca del 10\% del total de linfedemas ${ }^{(8)}$.

Secundario: se asociada entre otros a cáncer, radiación y cirugía ${ }^{(27,28)}$.

\section{Según la fase en que se encuentra}

Latencia: posterior a tratamiento, no se produce alteración. Puede durar años ${ }^{(27)}$.

Edema intermitente: secundaria a ciertas condiciones: sol, esfuerzo, traumatismo, episodios infecciosos. Se caracteriza por regresar por la mañana ${ }^{(27)}$.

Edema fijo: al inicio el edema es blanco blando e indoloro, con el signo de fóvea. Posteriormente la fóvea desaparece ${ }^{(27)}$.

Edema duro y tenso: el aumento local de proteínas y las complicaciones infecciosas, conducen a una fibrosis cutánea y una elefantiasis. Piel engrosada. A medida que el linfedema avanza se desarrolla una fibrosis dérmica ${ }^{(27)}$.

\section{Según su severidad}

Leve (grado 1): la diferencia en la circunferencia es de 2 a $3 \mathrm{~cm}$ y no hay cambios en los tejidos. En términos de volumen, existe una diferencia de 150 a $400 \mathrm{ml}(10-25 \%)$ en relación al lado sano. Edema mínimo.

Moderado (grado 2): La diferencia en la circunferencia es de 4 a $6 \mathrm{~cm}$. Un volumen con una diferencia de 400 a $700 \mathrm{ml}$ (26-50\%). Existen cambios definitivos en los tejidos, induración de la piel. Ocasionalmente erisipela. Edema moderado.

Grave (grado 3): Que se subdivide en dos:

3A: Diferencia mayor de $6 \mathrm{~cm}$ y de $750 \mathrm{ml} \mathrm{de}$ volumen total de diferencia. Entre el $50-100 \%$ de diferencia. Cambios acusados en la piel (queratosis, fístulas linfáticas o episodios repetidos de erisipela). Edema severo.

$3 B$ : Igual que $3 \mathrm{~A}$ pero hay dos o más extremidades afectadas. 
Elefantiasis (grado 4): Puede afectar varias extremidades, cabeza y cara. Más del 200\% de diferencia. Edema gigante.

\section{Complicaciones}

Como complicación del linfedema se puede encontrar:

- Disminución del arco de movimiento $\operatorname{articular}^{(6)}$.

- Infección de tejido subcutáneo y cutáneo ${ }^{(6)}$

- Problema cosmético ${ }^{(6)}$.

- Plexopatía del miembro superior ${ }^{(6)}$.

- Capsulitis adhesiva del hombro, también conocida como hombro congelado, con limitación en todos los arcos de movimiento del hombro (flexión, extensión, abducción y rotación) $)^{(7)}$.

- Linfangiosarcoma (Síndrome de Ste-

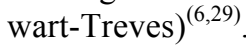

- Deterioro de la calidad de vida: Se ha reportado que la calidad de vida en las pacientes con linfedema es menor que en las pacientes sin éste ${ }^{(14)}$.

\section{Tratamiento}

El tratamiento del linfedema es difícil, costoso y consume tiempo. Es necesario un manejo interdisciplinario $^{(4)}$. Dentro del equipo de manejo es necesario incluir un especialista en Medicina Física y Rehabilitación, ya que tienen una visión global de la condición de las pacientes: problemas físicos, dimensión psicológica, integración social y profesional, calidad de vida, rehabilitación vocacional, es decir, pueden hacer diagnóstico, evaluación y manejo con la prescripción de terapia física y educación ${ }^{(19)}$.

Es necesaria la evaluación fisiátrica temprana en estas pacientes; idealmente realizarla prequirúrgicamente y debe incluir medidas de base de arcos de movimiento, diámetro, fuerza muscular e identificar posibles factores de riesgo y que así oriente al tratamiento adecuado. Ya que la rehabilitación es vital en la recuperación de las pacientes con CM, si es apropiado y a tiempo, porque puede prevenir complicaciones posquirúrgicas tempranas que pueden retrasar el tratamiento curativo del CM. La evaluación por los rehabilitadores de forma posquirúrgica debería ser previa a los tratamientos adyuvantes. Y el seguimiento-monitoreo por un servicio de Rehabilitación es necesario para identificar de forma precoz y manejar las complicaciones o limitaciones físicas asociadas al tratamiento del CM, lo cual mejora los resultados ${ }^{(12)}$.

No hay un consenso de un tratamiento específico para el linfedema, pero se recomienda la asociación de terapias ${ }^{(30)}$. No existen protocolos terapéuticos claros $^{(16)}$. Pero hay autores que consideran que la primera línea de tratamiento en el CM es la terapia física temprana prescrita por el especialista en rehabilitación ${ }^{(19)}$.

El objetivo primario del tratamiento del linfedema es facilitar el transporte linfático ${ }^{(31,32)}$. Hodgson et $a l{ }^{(33)}$ proponen 5 áreas prioritarias de abordaje: educación, investigación, prevención, normativa y promoción. El tratamiento habitual es la terapia física compleja (TFC) ${ }^{(16)}$.

\section{Automasaje}

Son todas las actividades que los individuos inician y realizan por sí mismos sin la ayuda de otros, no incluye los tratamientos administrados por los profesionales ${ }^{(34)}$.

\section{Presoterapia:}

Es una técnica de tratamiento por compresión externa, normalmente aire (compresión neumática). El aumento de la presión que producen los dispositivos de presoterapia lleva consigo un incremento del paso del líquido del intersticio al espacio vascular ${ }^{(35)}$.

No hay consenso en cuanto a cómo utilizar la presoterapia ni de los valores de presión a aplicar y la duración de la aplicación tanto en la sesión de tratamiento como en el número de sesiones. Como terapia aislada no es más efectiva que otras técnicas $^{(35)}$. La FDA aprobó el uso de las bombas de presoterapia de compresión neumática en $1976^{(6)}$.

\section{Drenaje linfativo manual (DLM)}

Es un masaje con una suave aplicación de presión sobre la piel y sigue la localización anatómica de los vasos linfáticos. Consiste en unas secuencias de determinados movimientos como los llamados círculos estacionarios, movimientos giratorios $^{(6)}$. Es lento e indoloro, se ha de ejercer escasa presión $(<40 \mathrm{mmHg})$ siguiendo un orden 
de maniobras centrífugo (desde la raíz a la zona distal) pero centrípeta en la aplicación de cada una de ellas ${ }^{(6)}$.

\section{Cinesiterapia (ejercicios de moviliza- ción de la extremidad)}

Para Eickmeyer ${ }^{(36)}$ la actividad física se debe describir como cualquier movimiento del cuerpo que requiera un gasto energético; puede ser una actividad de vida diaria o recreativa. Mientras que el ejercicio se define como el movimiento del cuerpo pero que es más estructural y tiene parámetros medibles como la grasa corporal, el rango de movimiento, la fuerza muscular y la capacidad cardiaca.

El ejercicio hace que se desarrollen vasos linfáticos colaterales, además del impacto positivo en el sistema muscular (efecto de bomba) y cardiovascular, que colaboran en mejorar el aclaramiento de la linfa ${ }^{(37)}$.

Para Stout ${ }^{(38)}$, antes de desarrollar un programa de rehabilitación e idealmente antes de la cirugía; se debería conocer el nivel premórbido de la función, los hábitos de ejercicio actuales, impedimentos físicos anteriores y otras comorbilidades para así poder establecer una línea de base de la pacientes y detectar cambios en el tiempo. Concretamente, el rango de movimiento de la extremidad superior, el volumen y la fuerza, el peso corporal, la función, la fatiga, y el nivel de actividad física y el ejercicio deben ser evaluados en la visita preoperatoria.

\section{Drenaje postural}

Se realiza mediante la elevación de la extremidad, para reducir la presión hidrostática intravascular y así, disminuir la producción de líquido linfático. Los requisitos posturales son una incomodidad, lo que supone un difícil cumplimiento del tratamiento ${ }^{(6)}$.

\section{Prendas/Vendajes}

Los vendajes persiguen el mismo objetivo y resultan más confortables, puesto que la presión que ejercen es menor. La superposición de varios vendajes (multicapa) aumenta el flujo de líquido linfático. Por otra parte, actúan como una medida de protección de la extremidad afectada frente a posibles traumatismos, heridas o quemaduras ${ }^{(16)}$.

\section{Autocuidado y cuidado de la piel}

"El autocuidado requiere tanto del aprendizaje como del uso del conocimiento, así como reforzar la técnica y motivación. El proceso de aprendizaje incluye el desarrollo gradual de un repertorio de prácticas de autocuidado y habilidades relacionadas",(28).

\section{Manejo del dolor}

El dolor se produce como consecuencia de la presión en terminales nerviosas o de contracciones musculares al moverse.

\section{Cirugía}

El tratamiento quirúrgico se utiliza para el edema irreversible $^{(6)}$. Se puede realizar microcirugía, transferencia ganglionar microquirúrgica y derivación microquirúrgica linfaticovenosa ${ }^{(26)}$.

\section{Farmacológico}

Puede usarse las benzopironas (más conocidas comúnmente como cumarinas) y otros compuestos de acción similar. Los tratamientos son prolongados y requieren determinación periódica de las enzimas hepáticas por su riesgo de hepatotoxicidad $^{(39)}$. Los vasopresores hacen que aumente de la presión de filtración y los diuréticos no movilizan proteínas de alto peso molecular, aumentando así la viscosidad ${ }^{(27)}$. Aunque hay autores como Hayes ${ }^{(40)}$ que han encontrado que no hay evidencia suficiente que soporte el uso de esos medicamentos.

\section{Medidas psicológicas}

Los repetidos fracasos del tratamiento, junto con las deformaciones físicas que suelen producir los linfedemas hacen a la paciente susceptible de sufrir depresiones, psicosis y complejos. Es importante explicar a las pacientes el posible proceso evolutivo y debe ser consciente de que se trata de una terapia paliativa y no curativa ${ }^{(6)}$.

No se ha encontrado evidencia científica sobre la utilización de electroterapia, laserterapia, hidroterapia o el uso de fármacos para la prevención primaria del linfedema ${ }^{(24)}$. 


\section{Prevención del linfedema}

Lo ideal es prevenir el linfedema y para ello existen diferentes métodos según el tipo de prevención $^{(16)}$ :

Prevención primaria: Es la realizada antes de que aparezca la enfermedad.

Prevención secundaria: Incluye los programas de detección precoz y medidas de profilaxis en las pacientes que tengan factores de riesgo.

Prevención terciaria: Se lleva a cabo una vez diagnosticado el linfedema instaurando un tratamiento para restablecer la normalidad, mejorar los síntomas y evitar el agravamiento clínico, aunque una vez instaurado como tal ya no tiene cura.

\section{CONCLUSIONES Y RECOMENDACIONES}

El linfedema es una patología frecuente en las pacientes de CM tratadas que con una adecuada rehabilitación se puede prevenir y en ocasiones revertir mejorando así sustancialmente la calidad de vida.

Se debe remediar la disparidad que existe en las referencias a los Servicios de Rehabilitación, ya que $22.4 \%$ de las pacientes no recibieron tratamiento rehabilitador y esto es aún peor en las poblaciones minoritarias y con desventaja socioeconómica $^{(38)}$.

La instauración de programas de prevención permite analizar de forma precoz el riesgo individual de padecer linfedema y en consecuencia diseñar una actitud terapéutica y un tratamiento precoz ${ }^{(28)}$. Por lo que se recomienda la referencia oportuna a los servicios de Rehabilitación de las pacientes mastectomizadas para la adecuada prevención y/o tratamiento del linfedema, independientemente de su estadio tumoral. El ejercicio físico por sí solo no es capaz de recuperar totalmente la funcionalidad del miembro superior $^{(45)}$.

Las pacientes identifican a la educación como la clave para desarrollar conciencia, fomentar el interés en la investigación y la promoción de las mejores prácticas en la atención clínica, imple- mentando modelos de rehabilitación o de enfermedades crónicas ${ }^{(22)}$.

\section{REFERENCIAS BIBLIOGRÁFICAS}

1. Varela E Lanzas G Atín MA González MV. Capítulo I: generalidades de los linfedemas y de la circulación linfática: patogenia y fisiopatología. Rehabilitación. octubre de 2010;44:2-7.

2. Castro A Moreno C Matarán G Aguilar M Almagro I Anaya J. Prevención del linfedema tras cirugía de cáncer de mama mediante ortesis elástica de contención y drenaje linfático manual: ensayo clínico aleatorizado. Med Clínica. 2011;137(5):204-207.

3. Fife CE Davey S Maus EA Guilliod R Mayrovitz HN. A randomized controlled trial comparing two types of pneumatic compression for breast cancer-related lymphedema treatment in the home. Support Care Cancer. 2012;20(12):3279-3286.

4. Gurdal SO Kostanoglu A Cavdar I et al. Comparison of Intermittent Pneumatic Compression with Manual Lymphatic Drainage for Treatment of Breast Cancer-Related Lymphedema. Lymphat Res Biol. 2012;10(3):129-135.

5. Mairena J. Impacto del cáncer de mama [Internet]. Caja Costarricense del Seguro Social; Octubre-13. Recuperado a partir de: http://www.ccss.sa.cr/noticias/index/arc hivo/29/1105-impacto-del-cancer-demama

6. Memorias del Symposium Zyma sobre Linfedema. V Congreso Panamericano de Flebología y Linfología. Ed. ZYMA S.A. Barcelona 1993.

7. Anderson RT Kimmick GG McCoy TP et al. A randomized trial of exercise on well-being and function following breast cancer surgery: the RESTORE trial. J Cancer Surviv. 2011;6(2):172181.

8. Arias A Álvarez MJ Martín R VillarinoDíaz C. Clínica, clasificación y estadiaje del linfedema. Rehabilitación. 2010;44:29-34.

9. Schmitz KH Speck RM Rye SA DiSipio T Hayes SC. Prevalence of breast cancer treatment sequelae over 6 years of 
follow-up: The Pulling Through Study. Cancer. 2012;118(S8):2217-2225.

10. Devoogdt N Van Kampen M Geraerts I Coremans T Christiaens MR. Lymphoedema Functioning, Disability and Health Questionnaire (Lymph-ICF): Reliability and Validity. Phys Ther. 2011;91(6):944-957.

11. Forner I Cuello E Forner A. Linfedema: diagnóstico diferencial y pruebas complementarias. Rehabilitación. 2010;44:14-20.

12. McNeely ML Binkley JM Pusic AL Campbell KL Gabram S Soballe PW. A prospective model of care for breast cancer rehabilitation: Postoperative and postreconstructive issues. Cancer. 2012;118(S8):2226-2236.

13. Bae H Kim HJ. Clinical Outcomes of Extracorporeal Shock Wave Therapy in Patients With Secondary Lymphedema: A Pilot Study. Ann Rehabil Med. 2013;37(2):229-234.

14. Park JE Jang HJ Seo KS. Quality of Life, Upper Extremity Function and the Effect of Lymphedema Treatment in Breast Cancer Related Lymphedema Patients. Ann Rehabil Med. 2012;36(2):240-247.

15. Jeong HJ Sim YJ Hwang KH Kim GC. Causes of Shoulder Pain in Women with Breast Cancer-Related Lymphedema: A Pilot Study. Yonsei Med J. 2011;52(4):661-667.

16. López M Valencia FJ González R Rodríguez FJ Crespo $\mathrm{P}$ Hernández MA. Validación de herramienta informática para el cálculo de linfedema en pacientes con afectación unilateral de extremidad superior. Rehabilitación. abril de 2011;45(2):127-133.

17. Devoogdt N Christiaens MR Geraerts I et al. Effect of manual lymph drainage in addition to guidelines and exercise therapy on arm lymphoedema related to breast cancer: randomised controlled trial. BMJ. 2011;343(1):d5326-d5326.

18. Forner C. Procedimientos diagnósticos en el linfedema. Angiología. 2010;62: 89.

19. Fialka-Moser V Korpan M Varela E et al. The role of physical and rehabilitation medicine specialist in lymphoedema. Ann Phys Rehabil Med. 2013;56(5):396-410.
20. Cuello E Forner I Forner A. Linfedema: métodos de medición y criterios diagnósticos. Rehabilitación. 2010;44:2128.

21. Loudon A Barnett $\mathrm{T}$ Piller $\mathrm{N}$ Immink MA Visentin D Williams AD. The effect of yoga on women with secondary arm lymphoedema from breast cancer treatment. BMC Complement Altern Med. 2012;12(1):66-75.

22. Norman SA Localio AR Kallan MJ et al. Risk Factors for Lymphedema after Breast Cancer Treatment. Cancer Epidemiol Biomarkers Prev. 2010;19(11): 2734-2746.

23. Demark-Wahnefried W Campbell KL Hayes SC. Weight management and its role in breast cancer rehabilitation. Cancer. 2012;118(S8):2277-2287.

24. González J Sánchez F. Calidad de vida, escalas de valoración y medidas preventivas en el linfedema. Rehabilitación. 2010;44:44-48.

25. Newman B Lose F Kedda MA et al. Possible Genetic Predisposition to Lymphedema after Breast Cancer. Lymphat Res Biol. 2012;10(1):2-13.

26. Masías J. Tratamiento microquirúrgico combinado para el linfedema de extremidades. Angiológía. 2010;62:90.

27. Brennan M DePompolo R. Focused Review: Postmastectomy Lymphedema. Arch Phys Med Rehabil. 1996;77:S74S80.

28. Armer JM Henggeler MH Brooks CW Zagar E. The Health Deviation of PostBreast Cancer Lymphedema: Symptom Assessment and Impact on Self-Care Agency. Self Care Depend Care Nurs. 2008;16(1):14-21.

29. Schmitz KH Troxel AB Cheville A et al. Physical activity and lymphedema (the PAL trial): Assessing the safety of progressive strength training in breast cancer survivors. Contemp Clin Trials. 2009;30(3):233-245.

30. De Fátima GM. Synergic Effect of Compression Therapy and Controlled Active Exercises Using a Facilitating Device in the Treatment of Arm Lymphedema. Int J Med Sci. 2012;9(4):280-284.

31. Tan IC Maus EA Rasmussen JC et al. Assessment of Lymphatic Contractile Function After Manual Lymphatic 
Drainage Using Near-Infrared Fluorescence Imaging. Arch Phys Med Rehabil. 2011;92(5):756-764.e1.

32. Ridner SH Bonner CM Deng J Sinclair VG. Voices From the Shadows: Living With Lymphedema. Cancer Nurs. 2012;35(1):E18-E26.

33. Hodgson P Towers A Keast DH Kennedy A Pritzker R Allen J. Lymphedema in Canada: a qualitative study to help develop a clinical, research, and education strategy. Curr Oncol Tor Ont. diciembre de 2011;18(6):e260-e264.

34. Ridner SH Fu MR Wanchai A Stewart BR Armer JM Cormier JN. SelfManagement of Lymphedema: A Systematic Review of the Literature From 2004 to 2011. Nurs Res. 2012;61(4): 291-299.

35. Cátedra E García M Puentes AB. Drenaje linfático manual y presoterapia. Rehabilitación. octubre de 2010;44:6367.

36. Eickmeyer SM Gamble GL Shahpar S Do KD. The Role and Efficacy of Exercise in Persons With Cancer. PM\&R. noviembre de 2012;4(11):874-881.

37. Schmitz KH. Balancing Lymphedema Risk: Exercise Versus Deconditioning for Breast Cancer Survivors. Exerc Sport Sci Rev. 2010;38(1):17-24.

38. Stout NL Binkley JM Schmitz KH et al. A prospective surveillance model for rehabilitation for women with breast cancer. Cancer. 2012;118(S8):21912200.

39. Belmonte R Tejero M Ferrer $\mathrm{M}$ et al. Efficacy of low-frequency low-intensity electrotherapy in the treatment of breast cancer-related lymphoedema: a crossover randomized trial. Clin Rehabil. 2011;26(7):607-618.

40. Hayes SC Johansson K Stout NL et al. Upper-body morbidity after breast cancer: Incidence and evidence for evaluation, prevention, and management within a prospective surveillance model of care. Cancer. 2012;118(S8):2237-2249.

\section{DECLARACIÓN DE CONFLICTO DE IN- TERESES}

Los autores declaran que no existen conflictos de intereses. 